踝 
Stadtforschung aktuell Band 60

Herausgegeben von:

Hellmut Wollmann 
Kirk Mildner

\section{Lokale Politik und Verwaltung in Russland}

Zwischen Neuanfang, Erbe und Korruption

Birkhäuser Verlag

Basel · Boston · Berlin 
Der Autor:

Dr. Kirk Mildner, geb. 1969, M.Litt., Dipl. Pol. hat in Berlin, St. Petersburg und St. Andrews Politologie, Osteuropawissenschaften und Internationale Beziehungen studiert. Derzeit ist er stellvertretender Leiter der Koordinierungsstelle der Kreditanstalt für Wiederaufbau in der deutschen Botschaft Moskau und beschäftigt sich mit der Koordinierung des wirtschaftlichen Beratungsprogrammes der Bundesregierung (Transform) für Russland.

Die Deutsche Bibliothek - CIP-Einheitsaufnahme

Mildner, Kirk:

Lokale Politik und Verwaltung in Russland : Zwischen Neuanfang, Erbe und Korruption/ Kirk Mildner. - Basel ; Boston ; Berlin : Birkhäuser, 1996

(Stadtforschung aktuell ; Bd. 60)

ISBN 978-3-8100-2792-4

ISBN 978-3-322-91365-4 (eBook)

DOI 10.1007/978-3-322-91365-4

Dieses Werk ist urheberrechtlich geschützt. Die dadurch begründeten Rechte, insbesondere die der Übersetzung, des Nachdrucks, des Vortrags, der Entnahme von Abbildungen und Tabellen, der Funksendung, der Mikroverfilmung oder der Vervielfältigung auf anderen Wegen und der Speicherung in Datenverarbeitungsanlagen, bleiben, auch bei nur auszugsweiser Verwertung, vorbehalten. Eine Vervielfältigung dieses Werkes oder von Teilen dieses Werkes ist auch im Einzelfall nur in den Grenzen der gesetzlichen Bestimmungen des Urheberrechtsgesetzes in der jeweils geltenden Fassung zulässig. Sie ist grundsätzlich vergütungspflichtig. Zuwiderhandlungen unterliegen den Strafbestimmungen des Urheberrechts.

() 1996 Birkhäuser Verlag, Postfach 133, CH-4010 Basel, Schweiz

Camera-ready Vorlage durch den Autor erstellt

Gedruckt auf säurefreiem Papier, hergestellt aus chlorfrei gebleichtem Zellstoff. TCF $\infty$

Umschlaggestaltung: Markus Etterich, Basel

ISBN 978-3-8100-2792-4

987654321 


\section{Inhalt}

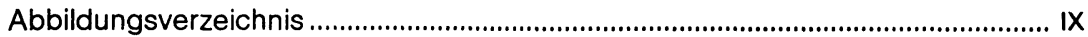

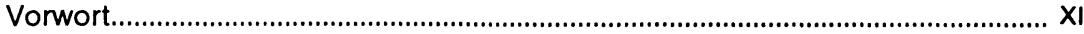

1. RuBlands Städte und Gemeinden im Aufbruch......................... 1

1.1 Gegenstand und Forschungsfragen .................................................... 1

1.2 Dimensionen und Determinanten institutionellen Wandels.................. 4

1.3 Forschungsmethodik und Auswahl der Fallbeispiele ........................... 10

2. Politische, gesellschaftliche und administrative

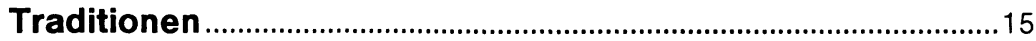

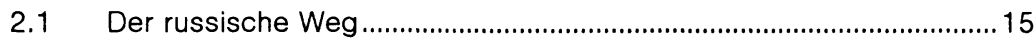

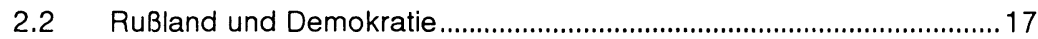

2.3 Die russische Rechtstradition: "načalo prava" versus "načalo vlasti"

2.4 Civil Society und Bürgertugend in RuBland......................................24

2.5 Bürokratietradition oder Korruption als System ..................................28

2.5.1. Sowjetbürokratie und systemische Korruption ...........................31

2.5.2. Funktionale Korruption? Die Lage im heutigen RuBland ..........36

3. Traditionen lokaler Selbstverwaltung in RuBland ......................43

3.1. Die Zemstvo-Bewegung ....................................................................4

3.1.1 Institutionen und Aufgaben der Zemstva ..................................45

3.1.2 Restauration, Belebung und Untergang .....................................46

3.2 Die russischen Gemeinden und Städte ..............................................49

3.3 Die Bauerngemeinden - Urkommunismus oder Modernisierungsbarriere?........................................................................52

3.4 Die lokale Ebene zwischen Samoderžavie und

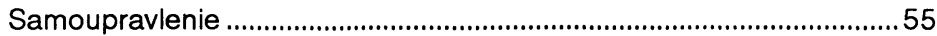

3.5 Kreise, Städte und Gemeinden unter der Sowjetherrschaft .................58

3.5.1 Lokale Sowjets und Wahlen .....................................................63

3.5.2 Strukturen der Sowjets und Lokalverwaltungen .........................65

3.5.3 Funktionen der lokalen Sowjets und deren Ispolkomy.............67

3.5.4 Totalitarismus oder Partizipation....................................................74

4. RuBlands Städte im Aufbruch: Die Entwicklung seit der Perestrojka ............................................................................. 76

$4.1 \quad$ Lokale Ebene und Perestrojka............................................................. 76

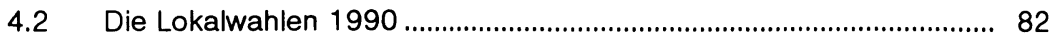


4.3 Die Eroberung der Exekutive: Die Entwicklung in Moskau und St. Petersburg ............................................................................................ 88

4.4 Das Selbstvenwaltungsgesetz vom 6. Juli 1991 .................................... 97

4.5 Doppelherrschaft und Desovetizacija bis zum Oktober 1993 ............. 102

4.5.1 El'cins Strategie für den lokalen ReformprozeB ......................... 103

4.5.2 Die Entwicklung des politisch-administrativen Systems bis Oktober 1993 am Beispiel des Kalininskij Rajons ............. 107

4.5.3 Entwicklungstendenzen der lokalen Ebene bis Ende 1993 im Vergleich

4.6 Oktober 1993: Die Neuordnung der lokalen (Selbst-)

Verwaltung.

5. Die Entwicklung seit Ende 1993 ..................................................123

5.1 Alle Macht der Verwaltung! .................................................................... 123

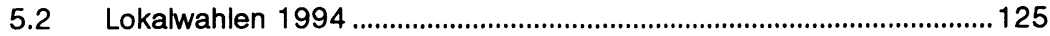

5.3 Die neuen Kommunalverfassungen ....................................................... 129

5.3.1 Ernennung oder Wahl der Bürgermeister .................................131

5.3.2 Die neuen lokalen Vertretungsorgane ..........................................134

5.3.3 Die Zukunft der Stadtrajons ......................................................137

5.3.4 Die Zukunft der Rajons .............................................................137

5.3.5 Die Zukunft der unteren Ebene der lokalen

Selbstverwaltung...........................................................................139

5.3.6 Übersicht zu einigen "Kommunalverfassungen"........................141

5.4 Das neue Selbstverwaltungsgesetz ...................................................146

6. Zur Entwicklung des lokalen Finanzsystems ............................152

6.1 Lokale Finanzen und Dezentralisierung............................................... 152

6.2 Das russische Steuersystem und lokale Einnahmen ............................ 154

6.2.1 Föderale und regionale Steuern ................................................154

6.2.2 Anteile der lokalen Ebene an föderalen und
regionalen Steuern ...................................................................158

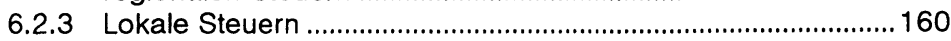

6.3 Übrige Einnahmequellen der lokalen Ebene ........................................165

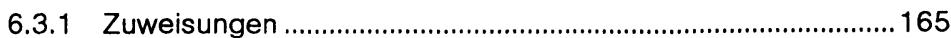

6.3.2 Gebühren und Abgaben .............................................................167

6.3.3 Einkünfte aus der Privatisierung und Vermietung
lokalen Eigentums ....................................................................168

6.3.4 Kredite und alternative Finanzierungsformen ...........................169

6.3.5 Außerbudgetäre Fonds ..........................................................170

6.4 Die Einnahmen der lokalen Ebene im Überblick................................... 174

6.5 Ausgaben der lokalen Ebene.............................................................. 175

6.6. Zusammenfassung............................................................................ 179 
7. Das lokale Wohnungswesen ...........................................................184

7.1 Veränderung der Rahmenbedingungen .............................................184

7.2 Wohnungsprivatisierung und Lokalverwaltungen .................................... 191

7.3 Mieten und Tarife: Zwischen Populismus und leeren Kassen .............195

7.4 Die Einführung von Wohngeld ............................................................202

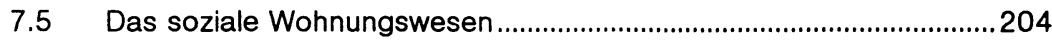

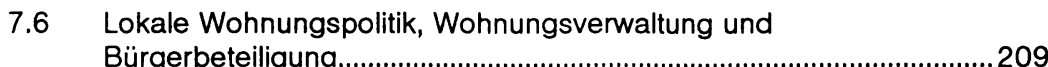

8. Lokale Wirtschaftsförderung und KMU's ....................................217

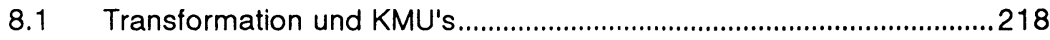

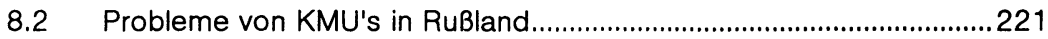

8.2.1 Kommunikationsprobleme........................................................222

8.2.2 Regelungskosten .....................................................................223

8.2.3 Bürokratische Obstruktion ......................................................224

8.2.4 Erpresserische Korruption.........................................................225

8.2.5 Das Fehlen intermediärer Institutionen .....................................226

8.3 KMU-Förderung: Programme und Institutionen ....................................226

8.3.1 Institutionen und Programme auf Zentralebene ........................227

8.3.2 Die Regionalebene ...................................................................2. 231

8.4 Wirtschaftsförderung auf lokaler Ebene ...............................................233

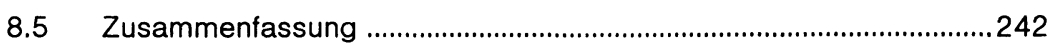

9. SchluBfolgerungen und Perspektiven..........................................244

9.1 Strukturen und konstitutionelle Fragen ..................................................244

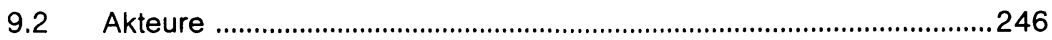

9.3 Aufgaben der lokalen Ebene und intergouvernementale

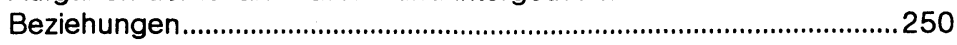

9.4 Lokale Demokratie, Bürgerbeteiligung und Kundenorientierung ...............................................................................2252

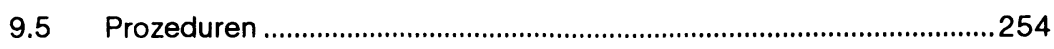

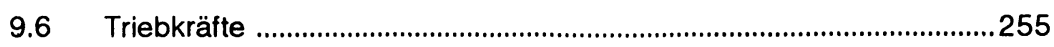

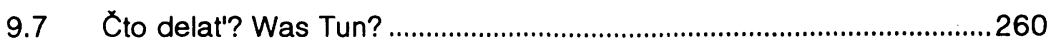

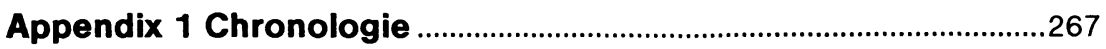

Bibliographie 


\section{Abbildungen}

Abb. 1.a Die 7-S-Matrix: Dimensionen des Wandels .............................................. 4

Abb. 1.b Dimensionen und Determinanten des institutionellen Wandels............ 9

Abb. 2. Übersicht der Fallbeispiele ................................................................. 11

Abb. 3. Aufgaben der Zemstva ..................................................................... 46

Abb. 4. Multisubordination der lokalen Ebene in der UdSSR ............................. 62

Abb. 5. Lokalverwaltung und Wohnungswesen ................................................... 71

Abb. 6. Abgeordnetenprofil des Leningrader Stadtsowjets................................ 84

Abb. 7. Die Lokalverfassung RuBlands nach dem Selbstverwaltungs-

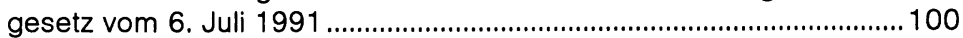

Abb. 8. Struktur des Kalininskij Rajonsowjets, Stand Juni 1992 ........................110

Abb. 9. Struktur der Kalininskij Rajonverwaltung, Stand Juni 1992 ...................112

Abb.10. Ausgewählte Ergebnisse der Lokalwahlen $1994 \mathrm{im}$ Leningrader Oblast' ................................................................................. 128

Abb.11. Lokalverfassung der Republik Komi ....................................................... 142

Abb.12. Präfekturverfassung der Stadt St. Petersburg........................................143

Abb.13. Lokalverfassung der Republik Udmurtija ................................................. 144

Abb.14. Die wichtigsten russischen Gesetze zum lokalen Finanzsystem

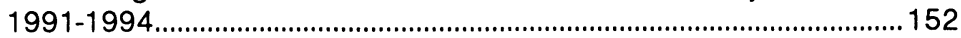

Abb.15. Steuerverteilung zwischen Föderation und Subjekten............................157

Abb.16. Steueranteile der Rajons und rajonfreien Städte 1994..........................158

Abb.17. Entwicklung der lokalen Anteile an den jeweiligen Steuern $1993 / 1994$

Abb.18. Steueranteile der rajonabhängigen Städte, Posëlki, Volosti und Stadtbezirke 1994

Abb.19. Lokale Bagatellsteuern in RuBland. 
Abb.20. Einnahmen durch Lokalsteuern

Abb.21. Zuweisungen im Gatčinskij Rajon 1994................................................166

Abb.22. Erfüllung veranschlagter Steuereinnahmen 1994_................................... 167

Abb.23. Normative Verteilung der Einkünfte aus der Privatisierung von munizipalem und föderalem Eigentum ..................................................... 169

Abb.24. Einnahmenstruktur von rajonfreien Städten und Kreisen ........................174

Abb.25. Einnahmenstruktur von rajonabhängigen Städten, Posëlki, Volosti und Stadtrajons.

Abb.26. Zuständigkeiten und Aufgabenmandate an die regionale und lokale Ebene.

Abb.27. Ausgaben rajonfreier Städte und Rajons 1993/1994

Abb.28. Ausgaben rajonabhängiger Städte, Posëlki, Volosti und Stadtrajons.

Abb.29. Fertiggestellter Wohnraum 1986-1993 .................................................186

Abb.30. Gesetzliche Neuordnung des Wohnungswesens ...................................189

Abb.31. Verhältnis zwischen Einkommen und Mieten im Vergleich ..................... 196

Abb.32. Ausgaben von Privathaushalten 1989-1993 ......................................... 197

Abb.33. Mieten auf dem freien Wohnungsmarkt in St. Petersburg.....................206

Abb.34. Das Wohnungswesen in Gatčina ...........................................................209

Abb.35. Institutionen der KMU-Förderung in Rußland.........................................22

Abb.36. Maßgebliche föderale Gesetze und Verordnungen für die Entwicklung von KMU's

Abb.37. Angewandte Instrumente der lokalen Wirtschaftsförderung ..................236

Abb.38. eziehungen zwischen Verwaltung und Bürgern .....................................252

Abb.39. Einnahmen von Städten, Kreisen und Gemeinden in OECDLändern

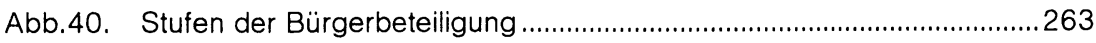

Abb.41. Taditionelle Verwaltung und New Public Management ..........................265 


\section{Vorwort}

Viele Personen haben am Entstehen dieser Arbeit mitgewirkt. Ihnen allen gilt mein aufrichtiger Dank!

Bei der Organisation und Durchführung der Feldforschung habe ich besonders Dr. Andrej Borisovič und Dr. Galina Gribanova von der Verwaltungshochschule in St. Petersburg, den ehemaligen Abgeordneten des Kalininskij Rajonsowjets Vladimir Sociev und Jurij Kočerevskij, Georgij Kolomenskij von der Stadtverwaltung in Gatčina, Dr. Natalija Butusova von der Universität Voronež und Dr. Valentina Kotel'nikova, Leiterin der Verwaltungshochschule der Republik Komi zu danken, ohne deren Hilfe das Projekt kaum durchzuführen gewesen wäre.

Mein Dank gilt außerdem Professor Hellmut Wollmann, Dr. Andreas HeinemannGrüder und Dr. Eckhard Schröter von der Humboldt-Universität Berlin, Silke Reinig, M.Litt von der Freien Universität Berlin und Ricarda Vulpius vom Osteuropa-Institut Berlin für ihre Expertise, ihre kritischen Anregungen und die Sorgfalt beim Lesen des Manuskriptes.

Es bedarf keiner Hervorhebung, daß die verbliebenen Fehler und Schwächen der Arbeit allein auf den Verfasser zurückfallen.

Gedankt sei schließlich der Friedrich-Naumann-Stiftung und dem Deutschen Akademischen Austauschdienst für die finanzielle Unterstützung des Projektes. 